\title{
High-sensitive C-reactive protein and fibrinogen as serological markers and predictors for the peripheral arterial diseases: Diagnosis and severity
}

\author{
Ayman M Samir,MD; Abdelrahman M Gameel,MD; Wael M Alsheemi,MD; \\ Mohamed A Azeez, MD
}

Vascular Surgery Unit, Faculty of Medicine, Zagazig University, Cairo, Egypt. Clinical Pathology Department, Faculty of Medicine, A/Azhar University, Cairo, Egypt.

\section{Co"espondence: E-mail:aymensamir@gmail.com}

\begin{abstract}
Purpose: To evaluate the role of the serological markers; hs-CRP and fibrinogen, in the prediction of vascular events in peripheral arterial diseases, and if it can be considered as prognostic risk factors for the severity of the atherosclerosis.

Methods: From February 2010 through March 2011, 200 subjects were included in this study; they consisted of 140 patients suffering peripheral arterial disease and 60 volunteers as controls. All patients were subjected to full history taking, measuring ABL duplex ultrasound and blood sampling to measure plasma levels of $h s-C R P$ and fibrinogen.

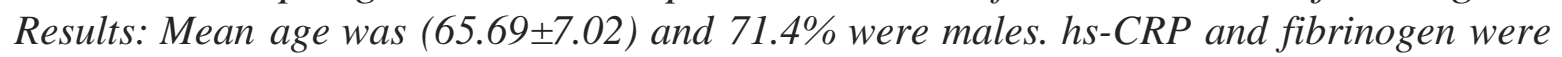
found highly significant risk factors for peripheral arterial disease prediction, and were also found inversely associated with ABL which means, they can be used as markers for disease progression or severity.

Conclusion: hs-CRP and fibrinogen are associated with increased risk of developing peripheral arterial disease and are considered as predictors of disease severity.
\end{abstract}

Introduction:

C-reactive protein (CRP) is a pentameric protein, which was discovered 70 years ago. I Historically, CRP has been used in the diagnosis and monitoring of active inflammation and infection.2 In mild inflammation and viral infection, CRP increases to $10-50 \mathrm{mgll}$. However, in active inflammation and bacterial infection, CRP concentration is $50-200 \mathrm{mg} / 1$. High concentrations are seen in sever infections.3 High sensitivity CRP techniques, using monoclonal and polyclonal antibodies (ELISA), can measure concentrations ofhigh sensitivity CRP as small as $0.15 \mathrm{mg} / 1.4$

The American Heart Association and Centers for Disease Control and Prevention (CDC) have identified 3 risk groups of people:

1- A low risk group with CRP concentrations less than $1 \mathrm{mg} / 1$.

2- A moderate risk group with CRP between 1 and $3 \mathrm{mg} / 1$.
3- A high risk group when CRP is higher than $3 \mathrm{mg} 11.5$

More recently, chronic inflammation has been identified as a component in the development and progression of atherosclerosis and CRP has been found to be a marker of cardiovascular risk.6 Recent studies showed that high concentrations within a normal range $(<5 \mathrm{mg} / 1)$ can predict future cardiovascular events.7

The role of the plasma fibrinogen as central protein in the coagulation system has been documented by clinical evidence: fibrous plaques are rich in fibrinogen and degradation products, which are involved in mechanisms of endothelial cell injury and platelet aggregation.8 It triggers the formation of progressive atherosclerosis plaques. The relationship between high plasma fibrinogen levels, the thin fibrous cap of atheroma, and a greater incidence of plaque rupture and thrombosis are well known. In patients with 
elevated fibrinogen levels, high numbers of inflammatory cells are concentrated in the shoulder and cap of plaques. 9

The aim of this research is to evaluate the role of the serological markers; high sensitive C-reactive protein ( hs-CRP) and fibrinogen, in the prediction of vascular events in peripheral arterial diseases, and if it can be considered as prognostic risk factors for the severity of the atherosclerosis.

\section{Patients and methods:}

This study was conducted at Vascular Surgery Unit, General Surgery Department, King Khalid Military Hospital, Saudi Arabia and at Vascular Surgery Unit, General Surgery Department, Zagazig University. We included 140 patients suffering peripheral arterial disease and 60 volunteers as control group. All patients were subjected to full history taking to determine the risk factors like age, smoking and systemic medical diseases. Thorough clinical examination was done to determine ankle brachial index (ABI) and the grade of chronic ischemia. Duplex ultrasound was done to all patients to confirm the diagnosis. Laboratory investigations which were done are blood glucose level, CRP (high sensitive) and plasma fibrinogen level. Routine laboratory values, urine analysis results and chest radiographs were used to exclude any inflammatory or infectious diseases.

Measurement ofhs-CRP was performed on the serum using an ultrasensitive assay based on a particle-enhanced turbidimetric immunoassay technique (Dade Behring). The assay was performed according to manufacturer instructions. Blood samples were mixed with the antiserum solution. The CRP reacts specifically with latex particles coated with antihuman CRP antibody to yield insoluble aggregates. The increase in turbidity that accompanies aggregation is proportional to the CRP concentration and was determined using an automated nephelometer.

Plasma fibrinogen activity was measured quantitatively by the Clauss method, where fibrinogen is converted from a soluble protein to an insoluble polymer by the action of thrombin, resulting in formation of a fibrin clot. The thrombin clotting time is inversely proportional to the fibrinogen concentration of the plasma. Thus, measuring the clotting time of dilute plasma when excess thrombin is added compared with a standardized fibrinogen preparation results in quantitative determination of fibrinogen concentration.

\section{Statistical analysis:}

Data were analyzed with SPSS 16.0. Values of continuous variables are expressed as mean \pm standard deviation. Groups were compared by the Mann-Witney Utest or matched signed rank test. Correlations between continuous variables were calculated using Spearman rank correlation coefficient Accuracy for prediction was additional assessed by receiver operating curve (ROC) analysis; thereby area under the curve (AUC) and optimal cutoff values of explanatory variables were calculated. All tests were considered significant at $\mathrm{P}<0.05$.

\section{Results:}

From September 2009 through March 2011, 200 subjects were included in this study; they consisted of 140 patients suffering of peripheral arterial disease and 60 controls of healthy individuals. Mean age was (65.69 \pm 7.02$)$, and $71.4 \%$ were males. Patients' and controls clinical characteristics are shown in Table(1) the age and sex were not statistically significant risk factors for PAD, while other risk factors \{Diabetes, hypertension, cardiac disease and smoking), high sensitive CRP and fibrinogen are highly significant risk factors for peripheral arterial disease. The statistical analysis using correlation coefficient and ROC curve showed that both hs-CRP and fibrinogen are sensitive and specific for diagnosis of peripheral arterial disease with more specificity and positive prediction value of hs-CRP. These data are shown in Table(2) and Figure(1), where the AUC and 95\% CI for hs-CRP is 0.96(0.980.94) when the hs-CRP level is more than or equal $2 \mathrm{mg} / \mathrm{dl}$, and for fibrinogen is $0.88(0.83$ 0.92 ) when the fibrinogen level is more than or equal $3.25 \mathrm{~g} / 1$. ABI was used as an indicator for disease severity, so on using correlation coefficient between ABI and hs-CRP and fibrinogen it was found that hs-CRP and fibrinogen were highly significant in prediction of disease severity, it is found that the high 
levels ofhs-CRP and fibrinogen are inversely proportion to the ABI as shown in Table(3).

About 90 of our patients underwent surgical or endovascular revascularization and it was fotmd that hs-CRP and fibrinogen plasma levels were reduced in about $72 \%$ of those patients, but we did not include these data inour results as it is out of the research purpose.

Table (1): Mean value of age,hs-CRP,fibrinogen and frequency of sex and risk factors among study patients and controL

\begin{tabular}{|l|c|c|c|c|c|l|}
\hline & \multicolumn{2}{|c|}{$\begin{array}{c}\text { Patients } \\
(\mathrm{N}=140)\end{array}$} & \multicolumn{2}{c|}{$\begin{array}{c}\text { Control } \\
(\mathrm{N}=60)\end{array}$} & $\begin{array}{c}\text { Test ofsig. } \\
\text { (t-test })\end{array}$ & P-value \\
\hline AgeX:I:SD & \multicolumn{2}{|c|}{65.697 .02} & \multicolumn{2}{|c|}{$63.73 \pm 9.0$} & 1.16 & 0.11 \\
\hline hs-CRP X:I:SD & $3.01 \pm 0.83$ & \multicolumn{2}{|c|}{$0.85 \pm 0.65$} & 17.8 & 0.000 \\
\hline Fibrinogen & $4.22 \pm 0.81$ & \multicolumn{2}{|c|}{$2.81 \pm 0.82$} & 11.24 & 0.000 \\
\hline Sex & No & $\%$ & No & $\%$ & XZ & \\
\hline \multicolumn{1}{|c|}{ Female } & 40 & 28.6 & 20 & 33.3 & 0.45 & 0.50 \\
\hline \multicolumn{1}{|c|}{ Male } & 100 & 71.4 & 40 & 66.7 & & \\
\hline Diabetes mellitus & 99 & 70.7 & 24 & 40.40 & 16.73 & 0.000 \\
\hline Hypertension & 100 & 71.4 & 24 & 40.0 & 17.6 & 0.000 \\
\hline Cardiac diseases & 85 & 60.7 & 20 & 33.3 & 12.6 & 0.000 \\
\hline Smoking & 80 & 57.1 & 24 & 40.0 & 4.94 & 0.02 \\
\hline
\end{tabular}

Table (2): Validity of fibrinogen, hs-CRP in dillgnosis of PAD.

\begin{tabular}{|l|c|c|c|c|c|}
\hline & AUC95\%CI & Sensitivity & Specificity & PPV & NPV \\
\hline Fibrinogen ( 3.25 gil) & $0.88(0.83-0.92)$ & 88.6 & 66.7 & 86.1 & 71.4 \\
\hline hs-CRP(2 mg/dl) & $0.96(0.94-0.98)$ & 82.1 & 86.7 & 93.5 & 67.5 \\
\hline
\end{tabular}

PPV: Positive predictive value

NPV: Negative predictive value

Table (3): Correlation coefficient between ABI, fibrinogen and hs-CRP.

\begin{tabular}{|l|c|c|}
\hline \multirow{2}{*}{} & \multicolumn{2}{|c|}{ ABI } \\
\cline { 2 - 3 } & $\mathrm{r}$ & $\mathrm{P}$ \\
\hline Fibrinogen & -0.79 & 0.000 \\
\hline hs-CRP & -0.28 & 0.001 \\
\hline
\end{tabular}

ROC Curve

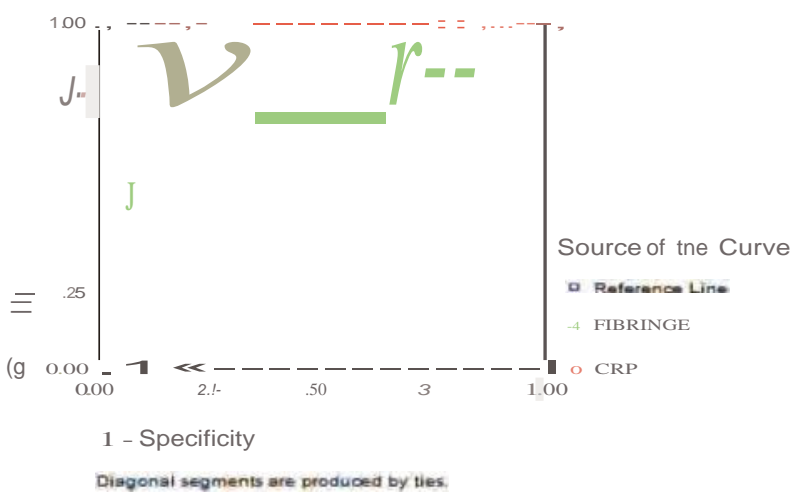

Figure (1): Sensitivity and specificity of fibrinogen and hs-CRP. 


\section{Discussion:}

Our study revealed that hs-CRP and fibrinogen are excellent serological markers for diagnosis of peripheral arterial disease and found statistically significant markers in correlation to the control group. On the other hand, these markers were found statistically significant with other risk factors (D.M., hypertension, cardiac diseases and smoking) for prediction of peripheral vascular diseases. Ridker et al,11 had reached the same results by comparing inflammatory markers in 14619 initially healthy American male physicians over a 9 year period. 140 of the participants developed PVD (peripheral vascular disease). This group was matched to a control group following age and risk factors. Hs-CRP was the strongest non lipid predictor of PVD.10,11 Also, in the Edinburgh artery study, a population of 1592 people were followed over a 12 year period. Hs-CRP was measured at baseline, 5 years and 12 years, and were correlated to the patients' symptoms and ABI. This study identified that hs-CRP is a useful predictor for PVD and its progression.I2 On the other hand, Musicant et al. did not show this strong association between D-dimer and CRP with peripheral arterial disease and concluded that these markers cannot be used as predictors for the disease. 13

Our results revealed high sensitivity and specificity of both hs-CRP and fibrinogen for diagnosis of peripheral arterial disease as demonstrated on (ROC) curve, especially if the hs-CRP level> $2 \mathrm{mg} / \mathrm{dl}$ and fibrinogen level $>3.25$ gll. Cassar et al., showed that hs-CRP levels were significantly higher in patients with PVD, 14 and $\mathrm{Vu}$ et al. concluded that patients with metabolic syndrome and hs-CRP higher than $3 \mathrm{mg} / \mathrm{L}$ have an increased risk ofPVD. 15

Heider et al. reported that elevated levels of fibrinogen and hs-CRP in patients with carotid artery stenosis versus controls imply that these acute-phase reactants are markers of plaque stability. Also, they found that elevated levels of these markers predict future risk of plaque rupture and ischemic stroke, particularly in men and in young and middleaged individuals. On the other hand, Heider et al., did not report any correlation between hsCRP and the prediction of the peripheral arterial disease.16 Levenson et al., reported that fibrinogen was elevated in subjects with silent atherosclerosis, particularly in those with disease in several arterial beds, which means that it is not specific for prediction of disease progression.I?

In the present study it was found that the levels of hs-CRP and fibrinogen are inversely proportion to the ABI, so, they can be markers of disease severity or the progression of the disease. McDormett et al. investigated that relationship between different inflammatory markers and $\mathrm{ABI}$ in patients with and without PVD, 370 patients with PVD $(\mathrm{ABI}<0.4)$ and 231 without PVD. ABI was significantly inversely associated with hs-CRP.18 Vainas et al. studied 387 PVD patients, at baseline and 12 months, looking for progression of the disease and future events. They concluded that hs-CRP was related to the severity of PVD and development of future cardiovascular events including death.19

It is important here to mention that the patients who underwent surgery, in our study, showed decreasing levels of fibrinogen and hs-CRP in postoperative period. The obvious question that arises when studying the effects of nonspecific markers of inflammation and thrombosis, such as hs-CRP and fibrinogen, is the clinical relevance of these associations. Our data suggest important role for use of these markers as initial screening tests for the presence ofPAD or for the risk of progression of symptomatic PAD.

\section{Conclusion:}

Our data demonstrate that elevated levels of the thrombotic and inflammatory markers, fibrinogen and hs-CRP, are associated with increased risk of developing peripheral arterial disease and are considered as predictors of disease progression or severity. It is, also, recommended to follow up the levels of these markers in postoperative period, after revascularization procedures, as reduction in their serum levels may reflect success in different lines of treatment, and helps in follow up of patients

\section{References:}

1- Khreiss T, JozefL, Potempa LA, Filep JG: Conformational rearrangement in $\mathrm{C}$ reactive protein is required for 
proinflammatory actions on human endothelial cells. Circulation 2004; I09: 2016-2022.

2- Yeh Edware TH: CRP as a mediator of disease. Circulation 2004; 109(Suppll): II-1-11-14.

3- Ledue TB, Rifai N:Preanalytic and analytic sources of variations inC-reactive protein measurement: Implications for cardiovascular disease risk assessment. Clin Chem 2004; 50(3): 574-581.

4- Abdellaoui A, Al-Khaffaf H: C-reactive protein (CRP) as a marker in peripheral vascular disease. Eur J Vase Endovasc Surg 2007; 34: 18-22.

5- Pearson TA, Mensah GA, Alexander WR, Anderson JL, Cannon RO, Criqui M, et al: Markers of inflammation and cardiovascular disease: Application to clinical and public health practice: A statement for healthcare professionals from the centers for disease control and prevention and the American Heart Association. Circulation 2003; 107: 499511.

6- Rifai N, Ridker PM: High-sensitivity Creactive protein: A novel and promising marker of coronary heart disease. Clin Chem 2001; 47: 403-411.

7- Albert CM, Ma J, Rifai N, Stamper MJ, Ridker PM:Prospective study of C-reactive protein, homocysteine, and plasma lipid levels as predictors of sudden cardiac death. Circulation 2002; 105: 2595-2599.

8- Bini A, Kudryk BJ: Fibrin and its derivatives in the normal and diseased vessel wall. Ann N Y Acad Sci 1992; 667: 112-126.

9- Mauriello A, Sangiorgi G, Palmieri G, Virmani R, Holmes DR Jr, Schwartz RS, et al: Hyperfibrinogenemia is associated with specific histocytological composition and complications of atherosclerotic carotid plaques in patients affected by transient ischemic attacks. Circulation 2000; 101: 744-750.

10-Ridker PM, Stampfer MJ, Rifai N: Novel risk factors for systemic atherosclerosis: A comparison of C-reactive protein, fibrinogen, homocystein, lipoprotein (a), and standard cholesterol screening as predictors of peripheral arterial disease.
JAMA 2001; 285:2481-2485.

11-Ridker PM, Cushman M, Stampfer MJ, et al: Plasma concentration of C-reactive protein and risk of developing peripheral vascular disease. Circulation 1998; 97: 425-428.

12-Tzoulaki I, Murray GD, Lee AJ, et al: Creactive protein, interleukin- 6 , and soluble adhesion molecules as predictors of progressive peripheral atherosclerosis in the general population: Edinburgh Artery Study. Circulation 2005; 112: 976-983.

13-Musicant S E, Taylor L M, Peters D, et al: Prospective evaluation of the relationship between C-reactive protein, D-dimer and progression of peripheral arterial disease. $J$ Vase Surg 2005; 43(4): 772-780.

14-Cassar K, Ford I, Greaves M, et al: Randomized clinical trial of the antiplatelet effects of aspirin-clopidogrel combination versus aspirin alone after lower limb angioplasty. Br JSurg 2005; 92 (2): 159165.

15-Vu JD, Vu JB, Pio JR, et al: Impact ofCreactive protein on the likelihood of peripheral arterial disease in United States adults with the metabolic syndrome, diabete mellitus, and pre-existing cardiovascular disease. Am J Cardio/2005; 96 (5): 655658.

16-Heider $\mathrm{P}$, Poppert $\mathrm{H}$, Wolf $\mathrm{O}$, et al: Fibrinogen and hig-sensitive C-reactive protein as serologic predictors for perioperative cerebral microembolic lesions after carotid endarterectomy. JVase Surg 2007; 449-454.

17-Levenson J, Giral P, Razavian M, et al: Fibrinogen and silent atherosclerosis in subjects with cardiovascular risk factors. Arterioscler. Thromb Vase Bio/1995; 15: 1263-1268.

18-McDermott MM, Greenland P, Guralnik JM, et al: Inflammatory markers, D-dimer, prothrombotic factors, and physical activity levels in patients with peripheral arterial diseases. Vase Med 2004; 9 (2): 107-115.

19-Vainas T, Stassen F, De GraafR, et al: Creactive protein in peripheral arterial disease: Relation to severity of the disease and to future cardiovascular events.J Vasc Surg 2005; 42 (2): 243-251. 